\title{
Energy Extraction and Achievement of the Saturation Limit in a Discharge-Pumped Table-Top Soft X-Ray Amplifier
}

\author{
J. J. Rocca, D. P. Clark, J. L. A. Chilla, and V. N. Shlyaptsev* \\ Department of Electrical Engineering, Colorado State University, Fort Collins, Colorado 80523
}

(Received 14 May 1996)

\begin{abstract}
We report the generation of laser pulse energies up to $30 \mu \mathrm{J}$ at $46.9 \mathrm{~nm}$ in the plasma column of a fast compressional capillary discharge. Double pass experiments using an iridium mirror achieved a gain-length product greater than 25 . The results provide the first clear evidence of saturated operation of a table-top soft x-ray amplifier. [S0031-9007(96)00858-7]

PACS numbers: 42.55.Vc, 52.55.Ez, 52.75.Va
\end{abstract}

A major goal in ultrashort wavelength laser research is the development of practical laser sources that can impact applications. Of particular interest is the demonstration of compact "table-top" amplifiers capable of generating soft x-ray pulses of substantial energy. Such development motivates the demonstration of gain media generated by compact devices that can be successfully scaled in length to reach gain saturation. At this condition, which occurs when the laser intensity reaches the saturation intensity, a large fraction of the energy stored in the laser upper level can be extracted. To date, gain saturation had only been achieved in a few soft $x$-ray laser transitions in plasmas generated by some of the world's largest laser facilities [1-4].

While gain in soft $\mathrm{x}$-ray lines has been reported in several compact systems [5-13] only in three cases the gain-length product was measured to be greater than 5 [5-7]. These include the observation of amplification at $46.9 \mathrm{~nm}$ in Ne-like Ar by our group in a plasma column generated by a fast capillary discharge [5,14], and the demonstration of gain at $41.8 \mathrm{~nm}$ in Pd-like $\mathrm{Xe}$ and at $32.6 \mathrm{~nm}$ in Ne-like $\mathrm{Ti}$ in plasmas generated by relatively compact terawatt class femtosecond and picosecond laser facilities, respectively [6,7]. In all cases, however, the laser energy extraction from table-top amplifiers has been small and detection of the laser lines was performed with high sensitivity detectors such as microchannel plate (MCP) intensifiers.

A next major step in the development of compact ultrashort wavelength lasers consists in advancing from gain observations to the demonstration of substantial laser output energies. Depending on the specific amplifier characteristics, this amounts to overcoming barriers that are imposed by small gain volumes and short plasma lengths, by short duration of the gain, or by axial plasma inhomogeneities and limiting refraction effects.

Recent optimization of the capillary discharge scheme, which has the advantages of a relatively large gain volume and long gain duration, allowed amplification at $46.9 \mathrm{~nm}$ to reach 14 gain-length products [15], but no demonstration of substantial laser energy extraction was realized. In this Letter we report the generation of laser pulse en- ergies up to $30 \mu \mathrm{J}$ at $46.9 \mathrm{~nm}$ in the plasma column of a fast compressional capillary discharge and the first clear evidence of gain saturation of a table-top soft x-ray amplifier. Single pass amplification experiments yielded laser pulse energies up to $6 \mu \mathrm{J}$. Greater energies were obtained by using an iridium mirror, in the first clearly successful double pass soft $\mathrm{x}$-ray amplification experiment in a discharge created plasma. These observations also demonstrate that these kinds of fast discharges can generate hot and dense plasma columns with aspect ratios approaching 1000:1 that have unprecedented axial uniformity and stability. This is of significance because previously plasma inhomogeneities due to nonuniform compression and plasma instabilities that destroy the amplification had hampered the development of discharge pumped soft $\mathrm{x}$-ray lasers [16]. We also report time resolved data that validate the results of magnetohydrodynamic calculations, giving a deeper understanding of the dynamics of the discharge plasma column and the lasing process.

The discharge setup and pulse generator used in the experiments have been previously described [17]. To conduct the double pass amplification experiments the anode electrode was modified to accommodate an iridium mirror in close proximity with the end of the plasma column. The measurements were conducted in polyacetal capillaries $4 \mathrm{~mm}$ diam filled with $700 \mathrm{mTorr}$ of pure Ar gas, and excited by current pulses of approximately $39 \mathrm{kA}$ peak current having a first half cycle duration of about $70 \mathrm{~ns}$. During the measurements conducted as a function of plasma column length, the amplitude and duration of the current pulses were maintained approximately constant by adjusting the discharge voltage and by equalizing the inductance in all discharges.

The results of single pass amplification measurements for capillary plasma columns up to $15.8 \mathrm{~cm}$ in length are shown as open circles in Fig. 1. A detection system consisting of a $2.2 \mathrm{~m}$ grazing incidence spectrograph with a MCP intensified array detector was used to measure the relative variation of the laser energy as a function of plasma column length. Calibrated Al filters of various thicknesses were used to avoid saturating the detector. The energy of the $46.9 \mathrm{~nm}$ laser line is observed to 


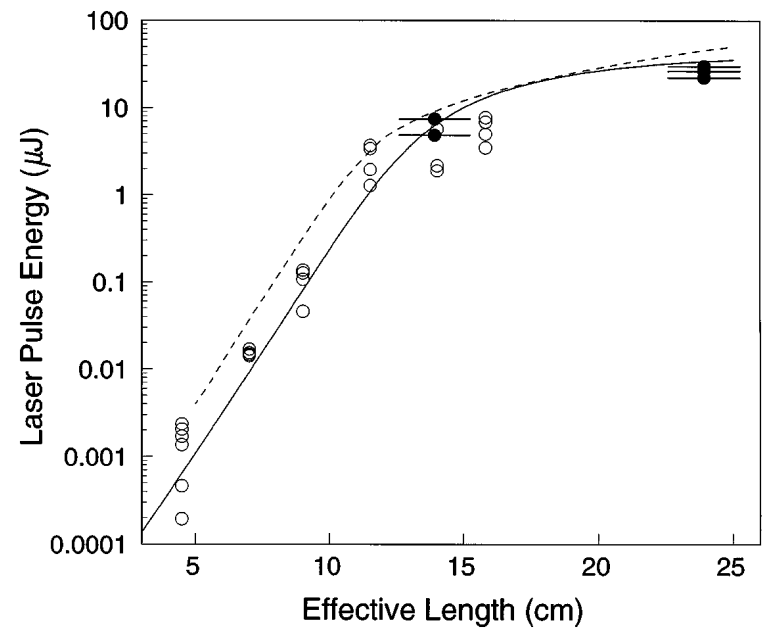

FIG. 1. Measured and computed $46.9 \mathrm{~nm}$ laser output energy as a function of capillary plasma column length. The single pass and double pass measurements are indicated by open and full circles, respectively. The relative energies were determined by using the spectrograph, with an intensified array detector and calibrated $\mathrm{Al}$ filters, and were scaled to the output energy of $6 \mu \mathrm{J}$ measured for the $15.8 \mathrm{~cm}$ long capillary by using the vacuum photodiode. The solid line is the result of simple radiation transport calculations assuming parabolic gain and density profile. The dashed line was computed using the full RADEX code.

increase exponentially for lengths up to $12 \mathrm{~cm}$, where it begins to saturate. A fit of the data corresponding to plasma column lengths up to $11.5 \mathrm{~cm}$ with the Linford formula [18] yields a gain coefficient $g=1.16 \mathrm{~cm}^{-1}$, a gain value that is close to that of previous measurements [15]. Saturation of the intensity is observed at a gainlength product of about 14 .

An absolute measurement of the laser pulse energy extracted in a single pass was conducted, and used to calibrate the data points in Fig. 1. For this measurement we used a fast vacuum photodiode having an $\mathrm{Al}$ photocathode. The quantum efficiency of the Al photocathode was determined to be $7.4 \%$ at $47 \mathrm{~nm}$ [19]. The photodiode was operated with a $1000 \mathrm{~V}$ bias to achieve a characteristic (10-90)\% rise time and an $R C$ fall time of $124 \mathrm{ps}$ and $23 \mathrm{ps}$, respectively. To conduct the measurements the photodiode was placed at approximately $60 \mathrm{~cm}$ from the laser after a $\mathrm{Cu}$ mirror. The diode signal was recorded in a $2 \mathrm{GHz}$ analog bandwidth digitizing oscilloscope [20]. Stainless steel meshes with fine grids were used to attenuate the laser beam power. Measurements were conducted as a function of bias voltage to verify that space charge and the limited photodiode rise time had a negligible effect in the recorded wave form at the employed voltage. As discussed later, the laser pulse is intense and short and clearly distinguished from the spontaneous emission background [see Fig. 3(a)]. A laser output energy of $6 \mu \mathrm{J}$ was determined for a $15.8 \mathrm{~cm}$ long capillary by integrating the photodiode signal and correcting for the absorption of the neutral Ar gas present between the discharge and the detector.

Double pass amplification experiments allowed us to substantially increase the laser pulse energy and to study the saturation behavior for effective plasma column lengths significantly longer than the maximum capillary length of $16 \mathrm{~cm}$ utilized in these experiments. Double pass amplification measurements were performed using flat iridium mirrors for two different plasma column lengths: $l=9$ and $14 \mathrm{~cm}$. The mirrors were fabricated by polishing vacuum melted Ir to a surface roughness better than $15 \AA$. The normal incidence reflectivity at $47 \mathrm{~nm}$ is about 20\% [21]. To minimize absorption by neutral $\mathrm{Ar}$ atoms the mirrors were placed in close proximity to the end of the plasma column, at $5 \mathrm{~mm}$ from the back surface of the anode electrode. Because of this close proximity with the plasma the mirrors were damaged by the discharge shots. Degradation occurs when the dense plasma reaches the mirror, about $1 \mu \mathrm{s}$ after the laser pulse. Nevertheless, in some cases up to three discharge shots could be performed before a significant degradation of the reflectivity was observed. To determine the enhancement caused by the mirrors, the laser intensity measured in the double pass amplification shots was compared with the intensity of reference shots without the mirror, performed immediately before and after each sequence of mirror shots. For $9 \mathrm{~cm}$ plasma columns the double pass intensity was measured to exceed by $63 \times$ the intensity of the single pass shots in the same capillary. In contrast, the laser intensity enhancement observed in the double pass experiments in $14 \mathrm{~cm}$ long capillaries was on average only $8 \times$. This behavior is indicative of saturation of the amplification in the second pass.

The energy of the double pass shots, full circles in Fig. 1, was obtained by scaling with the measured enhancements the output energy obtained for capillaries of the same length in a single pass. The corresponding effective amplification lengths were computed as $L_{e}=(2 g l+$ $\ln \eta) / g$, where $\eta$ is the feedback fraction from the mirror [3]. The values of $L_{e}$ were determined within an uncertainty range (horizontal bars) defined by the extreme values of $\eta$. The effective feedback fraction is significantly smaller than the $20 \%$ mirror reflectivity mainly due to the imperfect coupling between the modal area of the reflected beam and the cross sectional area of the gain region. It is also reduced by absorption of neutral Ar gas in the space between the plasma column and the mirror. The minimum value of $\eta$ was determined from the intensity enhancement measured in the $9 \mathrm{~cm}$ long plasma, and the maximum was estimated from the losses mentioned above considering the largest possible modal/gain area ratio. The effective length for the double pass experiment in the $14 \mathrm{~cm}$ long capillary is $23.9 \pm 1.3 \mathrm{~cm}$, corresponding to a maximum obtained gain-length product of $27.7 \pm 1.5$. The double pass data points in Fig. 1 are observed to be in 
good agreement with the saturation behavior observed in the single pass experiments.

The increase in the laser energy measured in the double pass experiments in the $14 \mathrm{~cm}$ columns corresponds to laser pulse energies up to $30 \mu \mathrm{J}$ and intensities larger than the computed saturation intensity of $56-78 \mathrm{MW} / \mathrm{cm}^{2}$. The saturation intensity was calculated considering an ion temperature of $100 \mathrm{eV}$, and an effective to radiative lifetime ratio for the laser upper level between 20 and 30 for plasma densities of $(5-8) \times 10^{18} \mathrm{~cm}^{-3}$, as computed by our magnetohydrodynamic model calculations for these discharge conditions. Such laser pulse energy is a significant fraction of the maximum energy that can be extracted in a $0.8 \mathrm{~ns}$ pulse from a $46.9 \mathrm{~nm}$ amplifier column $14 \mathrm{~cm}$ long by $300 \mu \mathrm{m}$ in diameter, having a gain of $1.2 \mathrm{~cm}^{-1}$, estimated to be about $100 \mu \mathrm{J}$.

The observed saturation of the gain and the measured laser pulse energy were compared with the result of simple radiation transport calculations for the capillary plasma column. Computations of the variation of the laser energy as a function of plasma column length were conducted by solving the radiation transport equation for an inhomogeneously broadened transition, taking into account line narrowing, refraction effects, and gain saturation. It should be noticed that consideration of refraction losses is essential to adequately describe the measured energy dependence on plasma column length. In these calculations the simplifying assumption of parabolic gain and density profiles [22] was adopted. The solid line in Fig. 1 shows the results of calculations performed for a plasma column having density and gain profiles of $300 \mu \mathrm{m}$ FWHM diameter and a plasma density of $8 \times 10^{18} \mathrm{~cm}^{-3}$. These plasma parameters are in reasonable agreement with those determined from hydrodynamic computations, and define a refraction length of $5.3 \mathrm{~cm}$ [22] and a corresponding refraction loss of $0.38 \mathrm{~cm}^{-1}$. The good agreement with the measurements confirms the saturated operation of the discharge-pumped amplifier. Calculations of the saturation behavior were also conducted for the time dependent electron density and gain profiles obtained from magnetohydrodynamics and atomic physics calculations. The computations were conducted with the code RADEX [23,24], using self-consistent non-local thermal equilibrium atomic physics in the transient approximation, with radial and axial radiation transport. The results, shown as a dashed line in Fig. 1, also indicate that gain saturation was reached. Further evidence is provided by independent experiments that allow us to discard refraction and duration of the gain as possible causes for the observed gain reduction for long capillaries. In the present study the observed beam profiles consistently displayed a single central peak without significant side lobes, as shown in Fig. 2(a). This indicates that in this experiment refraction effects [22] are not a limitation to the exponential growth of the laser intensity. The duration of the gain was
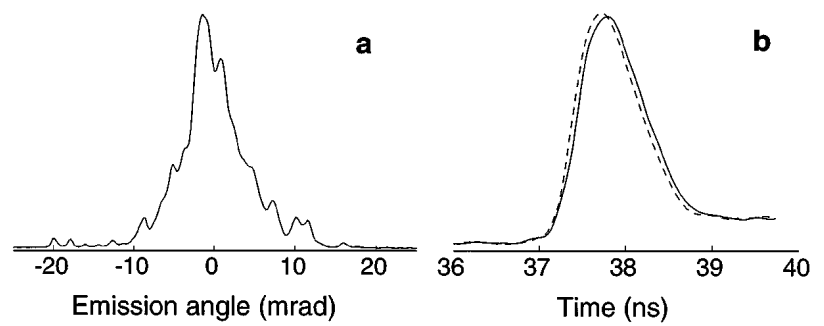

FIG. 2. (a) Cross section of the laser beam profile recorded at the focal plane of the spectrometer along the direction of the slit for a plasma column length of $16 \mathrm{~cm}$. (b) Time resolved measurement of the laser pulse. The solid line is as recorded; the dashed line is corrected for the response of the detection system.

obtained from photodiode measurements such as that shown in Fig. 2(b). The recorded signal was corrected for the limited bandwidth of the detection system. At the discharge conditions corresponding to Fig. 1 and for plasma columns $14 \mathrm{~cm}$ in length the FWHM pulse width was measured to be about $0.8 \mathrm{~ns}$. Because of the $1 / \sqrt{g l}$ dependence of the laser pulse duration, such pulse width is indicative of FWHM gain duration of more than $2 \mathrm{~ns}$. Such gain duration, which agrees with that obtained from our model calculations, exceeds the value that would limit the amplification to the observed plasma column saturation length of less than $15 \mathrm{~cm}$.

Finally, we discuss the observed temporal evolution of the on-axis radiation, shown in Fig. 3(a). The laser pulse is distinctively intense and narrow. It is followed approximately $6 \mathrm{~ns}$ later by a broader, less intense pulse corresponding to the spectrally integrated incoherent soft $\mathrm{x}$-ray plasma emission produced at the time of stagnation. These results were compared with our magnetohydrodynamics and atomic physics computations to obtain an increased understanding of the lasing process in relation with the evolution of the plasma column. The dynamics of the plasma column and of the laser pulse depend on the current distribution during the plasma compression. A significant part [(20-50)\%] of the current is computed to flow through material ablated from the walls by plasma radiation and heat conduction. Up to the time of lasing wall ablation by radiation is dominated by lines in the $40-80 \mathrm{~nm}$ region, corresponding to preneonlike ions. This is due to the larger abundance of lines in this spectral region, where the photoinization absorption of the wall material is larger [25], and to the larger radiation loss rates of the lower ionization stages [26]. Figure 3(b) depicts the computed temporal evolution of the intensity of the $46.9 \mathrm{~nm}$ amplified line emission and of the on-axis spontaneous emission. The calculated evolution of the electron temperature and density in the vicinity of the capillary axis is shown in Fig. 3(c). The optimum conditions for lasing occur several ns before stagnation, when the first compression shock wave reaches the axis. It can be seen that 


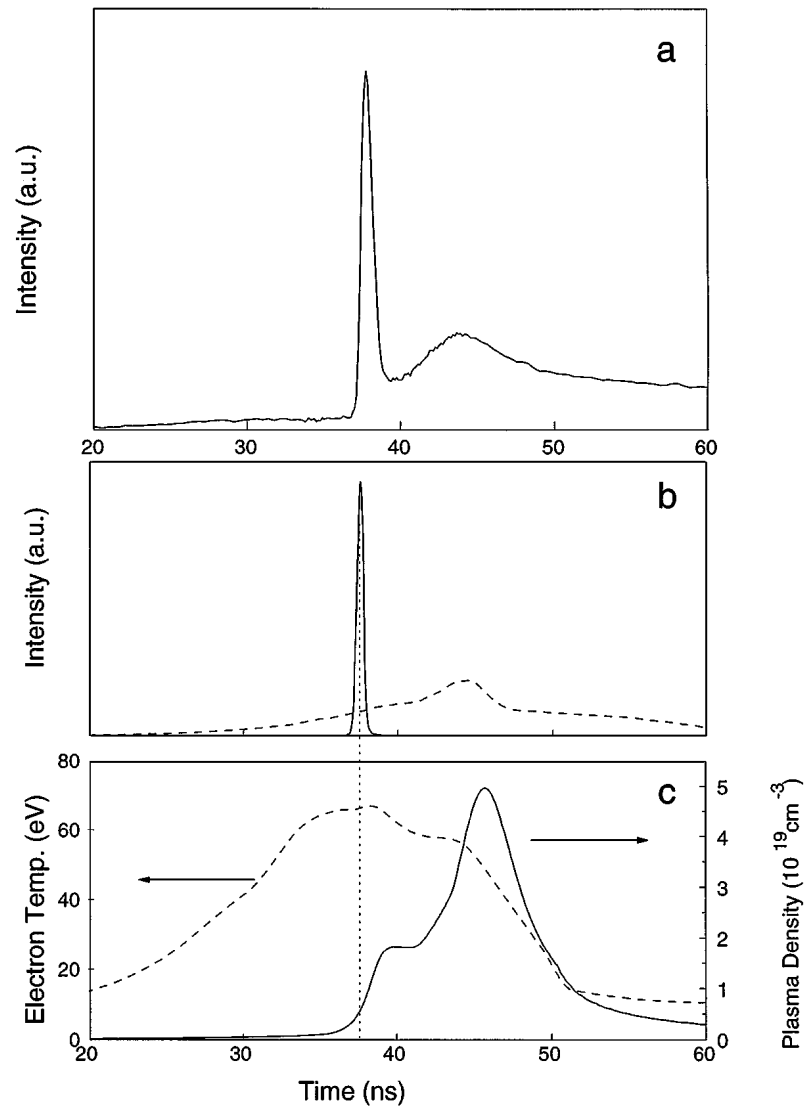

FIG. 3. (a) Measured evolution of the soft x-ray emission from a $14 \mathrm{~cm}$ long Ar capillary discharge. (b) Computed temporal evolution of the intensity of the $46.9 \mathrm{~nm}$ amplified line emission (solid line) and the spectrally integrated on-axis spontaneous emission (dashed line). (c) Computed temporal evolution of the electron density and temperature in the region within $100 \mu \mathrm{m}$ from the axis. The time is measured with respect to the beginning of the current pulse.

lasing takes place on the slope of the increasing electron density when it reaches values near $(0.3-1) \times 10^{19} \mathrm{~cm}^{-3}$ at the temperature of 60-80 eV. Subsequently, the electron density continues to increase as the plasma stagnates, and the laser action ceases due to increased refraction and collisional thermalization. The electron density and the spontaneous emission are computed to reach their peak values at stagnation, 5-8 ns after the laser pulse. The model results agree with the observed temporal behavior of the spontaneous emission and lasing pulse, giving a good description of the entire evolution of the plasma column and of the dynamics of the lasing process.

In conclusion, we have achieved laser pulse energies up to $30 \mu \mathrm{J}$ and have observed saturated operation of a $46.9 \mathrm{~nm}$ table-top discharge-pumped amplifier. This is the first demonstration of substantial energy extraction from a table-top soft x-ray amplifier. The above results are expected to lead to the development of very compact and practical ultrashort wavelength lasers that can be widely utilized in applications.
We thank B. Bach for manufacturing the mirrors and A. Osterheld for providing the atomic data. We also acknowledge the contributions of M. Marconi, F. Tomasel, and O. Cortazar. This work was supported by the NSF Grants No. ECS 9401952 and No. ECS 9412106. Part of the diagnostics instrumentation was developed in collaboration with Hyperfine Inc, Boulder, $\mathrm{CO}$, with support from the Colorado Advanced Technical Institute.

*On leave from P.N. Levedev Physics Institute, Moscow, Russia.

[1] B. J. MacGowan et al., Phys. Fluids B 4, 2326 (1992).

[2] J. A. Koch et al., Phys. Rev. Lett. 68, 3291 (1992).

[3] A. Carillon et al., Phys. Rev. Lett. 68, 2917 (1992).

[4] B. Rus et al., in X-ray Lasers 1994, edited by D. Eder and D. Matthews, AIP Conf. Proc. 332 (AIP, New York, 1994), p. 152; S. Wang et al., ibid., p. 293.

[5] J. J. Rocca et al., Phys. Rev. Lett. 73, 2192 (1994).

[6] B. Lemoff et al., Phys. Rev. Lett. 74, 1574 (1995).

[7] P. V. Nickles et al., SPIE J. 2520, 373 (1995).

[8] T. Wagner et al., Phys. Rev. Lett. 76, 3124 (1996).

[9] Y. Nagata, K. Midorikawa, and S. Kubodera, Phys. Rev. Lett. 71, 3774 (1993).

[10] S. Basu, P.L. Hagelstein, and J. G. Goodberlet, Appl. Phys. B 57, 303 (1993).

[11] C. O. Park, L. Polonsky, and S. Suckewer, Appl. Phys. B 58, 19 (1994).

[12] H.-J. Shin, D.-E. Kim, and T.-N. Lee, Phys. Rev. E 50, 1376 (1994).

[13] S. Glenzer and H.-J. Kunze, Phys. Rev. E 49, 1586 (1994).

[14] J. J. Rocca et al., Phys. Plasmas 2, 2547 (1995).

[15] J. J. Rocca et al., IEEE J. Sel. Top. Quantum Electron. 1, 945 (1995).

[16] R. C. Elton, X-Ray Lasers (Academic, Boston, 1990).

[17] J. J. Rocca et al., Phys. Rev. E 47, 1299 (1993).

[18] G. J. Linford et al., Appl. Opt. 13, 379 (1974).

[19] The quantum efficiency $\left(q_{e}\right)$ of our Al photocathode was obtained from comparison with an extreme ultraviolet silicon photodiode of known $q_{e}$. Using a He capillary lamp filtered with the spectrometer we measured a $q_{e}$ of $9.2 \%$ at $58.4 \mathrm{~nm}$. Correcting for the dependence of electron yield of Al photocathode on photon energy [27], the $q_{e}$ at $46.9 \mathrm{~nm}$ is $\sim 20 \%$ smaller: $7.4 \%$.

[20] Model HP 54720D with plug-in model HP 54722A.

[21] D. L. Windt, Ph.D. thesis, Physics Department, University of Colorado, Boulder, 1987.

[22] R. A. London, Phys. Fluids 31, 184 (1988).

[23] V. N. Shlyaptsev et al., SPIE J. 2012, 99 (1993).

[24] V. N. Shlyaptsev, J. J. Rocca, and A. L. Osterheld, SPIE J. 2520, 365 (1995).

[25] B. L. Henke, E. M. Gullikson, and J.C. Davis, At. Data Nucl. Data Tables 54, 181 (1993).

[26] A. V. Vinogradov and V.N. Shlyaptsev, Sov. J. Quantum Electron. 17, 1 (1987).

[27] R. H. Day et al., Los Alamos Technical Report No. LAUR-79-1360 (unpublished). 